as death. The physician was now somewhat alarmed, though he felt that the blood-letting had not been carricd too far, gave her one-twenty-fourth gr. morphia and ten grs. bromide pot., to be repeated every two hours until pain was relieved. She received two doses.

March 14, 9 A.M., patient had had some sleep; still complains of head, though more comfortable than on preceding day. Morphia and bromide to be continued until relieved. 6 P.M. applied blister over whole forehead to relieve pain, which has continued. Patient's general condition seems to be decidedly improved.

March 15,9 A.M. Pain in forehead much relieved. Slep well, pulse 88 , skin not so pale. Medicines were ordered discontinued unless pain returned.

March 15,9 A.M. Patient still improves, has commenced to take food (she took none whatever during these days of distress). Gave oil to move bowels. Applications of ice still continued because patient will not permit their removal.

March 17. Took away ice bags and directed cold water cloths to be used instead. Convalescence continued slowly and patient did not require further active treatment. On the 23d she said she felt very well, and was able to sit up a few days later. She made a good recovery.

\section{OUGHT INFANTS TO BE WASHED DIRECTLY AFTER BIRTH?}

Read in the Section of Diseases of Children, at the Forty-third Annual Meeting of the American Medical Association, held at Detroit, Mieh.. June. 1892.

BY FRANK S. PARSONS, M.D., OF NORTHAMPTON, MASS.

LECTURER ON DISEASES OF CHILDREN IN THE COLLEGE OF PHYSICIAN AND SURGEONS, BOSTON, MASS.

Doubtless most physicians find themselves confronted by this question nearly every week.

The dire results in some instances, where infants have been subjected to severe exposure to cold, during a prolonged process of this part of their toilet, would perhaps lead one to think a negative answer should be given. On the other hand, there are cases that have seemingly received the same treatment, and come out unscathed by any disease the etiology of which is credited to undue exposure to chilling influences.

There is, probably, no hygienic measure more tempting to a good nurse, than to arrange the toilet of the little one, making its body sweet and clean, as early as possible after birth.

If, as the custom is, we look to nature, as manifested in the lower animals, for a solution of this problem, we find that all the domestic, and so far as we are able to know the wild animals, wash their young directly after birth.

Of course there are many reasons why this fact, even though natural, can be no criterion to our treatment of the human race. The human mother cannot perform for her offspring such duties in the manner which the lower animals exhibit, nor would she be expected to were it a practical, or even fashionable thing to do. Then, too, the young of the lower animals being more thickly covered with short hairs or fur, the process with them is more one of drying the already wetted surface, than any particular desire for cleanliness. It is a natural instinct, or desire, on the part of the mother, to promote a healthy reaction of the skin of her offspring, who, in a wet condition, has suddenly been transferred from a higher temperature to one considerably lower.

With the human infant there is a different natural arrangement. A child has little, practically no, protecting hair. At birth it is suddenly ushered from an aqueous solution of nearly $100^{\circ} \mathrm{Fahrenheit,} \mathrm{to} \mathrm{an}$ aerial temperature from $20^{\circ}$ to $30^{\circ}$ colder. In other words, it has quickly been surrounded by a cold wave.

Then, if the infant is fortunate, it is received into some warmed flannel or woolen garment, and allowed to rest until the mother is amply cared for.

Now, while a baby has no constitutional environments for warmth and protection from exposing draughts, as has the animal, yet nature does provide an element of protection, seemingly for this emergency.

This is the sebaceous material consisting of fatty matter, epithelial cells, and other detritus found in greater or less quantity on the skin of the newborn child. This, if allowed, owing to the presence of the fatty matter, would naturally protect the infant from the chilling influences of reduced temperature to a considerable degree, and of itself become exfoliated about the time the umbilical cord separates.

However, the average nurse is imbued with the idea that, as soon as her other duties are over, the infant must be immediately washed, and turns her attention towards depriving it of this protecting element, by the application of soap and water.

Personally, I do not so much object to the washing of an infant directly after birth, as the manner in which it is commonly performed.

I trust I may be pardoned for occupying your time in rehearsing this part of the infant's toilet, as it is commonly done, and with which you all must be familiar, but I wish it contrasted with the method I shall hereafter recommend.

The baby, uncovered from head to foot, lies in the nurse's lap; she takes a soft sponge or rag, and with soap of a varying degree of alkalinity, and water of more or less warmth, washes small areas of the child at a time, and dries it with a towel or cloth of a greater or less degree of softness, until all stains and sebaceous matter are removed.

By the time this process is accomplished, and especially in cold months, the child may be seen shivering, and a bluish cast appearing over the exposed surface of its body.

Then the navel is hurriedly dressed, the infant attired in more or less suitable robes, and placed in bed with its mother to get warm.

Such is the treatment of a large per cent. of the children of to-day, and such are the cases that, almost invariably, develop catarrhal conditions varying in severity from simple snuffles to bronchopneumonia, with all its attending dangers.

I will not weary you with statistical references to cases of bronchitis, and other catarrhal affections, traceable to the first bath of infancy as a cause, as you all, doubtless, have had large experience in that line.

The question, then, is one of true infant hygiene. Should a newborn child be deprived of its natural protecting element? If so, what method should be employed to lessen the danger to the infant's health during the process?

If some little skirt with sleeves and hood could be contrived, made of some warm, unirritating material, such as canton flannel, in which the infant, after being quickly oiled with pure hog's lard, could be wrapped and allowed to remain four or five days, until it had become accustomed to the reduced temperature, and then properly washed, it would doubtless be a less exposing method of treatment.

This, however, will not suit the average mother, 
who wants her infant aweet and clean, and attired in all the paraphernalia of its wardrobe immediately after birth, so that it may be shown to the interested friends of the family.

This being an inevitable fact, it remains for us to devise some method of washing infants with the least exposure to cold, and for this reason, I have for some time suggested the following plan of treatment:

When there exists a large amount of sebaceous matter, ordinary soap and water will not remove it, so in these cases I direct the child to be previously anointed with pure hog's lard, this being the best solvent of the white sebum.

Then the child is quickly returned to the temperature from which it originally came by immersing it, to its neck, in a tub of water, the temperature of which is $103^{\circ}$ Fahrenheit, or thereabouts.

Then the child, supported on the arm of the nurse, is vith her other hand, or by the aid of an assistant, washed underneath the surface of the water, using only a soft linen rag or old handkerchief, the hem of which has been removed, and the best Castile soap.

Although not absolutely necessary, it is better, if possible, to have a second tub of water, equal in temperature to the first, in which the child may be rinsed.

This being accomplished, which should not have occupied more than five or six minutes, the child is quickly placed in a warmed absorbing blanket, and with the hand on the outside of the garment thoroughly but gently rubbed.

Afterwards the baby should be removed and rolled in a second warm and dry blanket, and allowed to remain a couple of hours, after which it may be dressed in garments suitable for comfort and protection.

I have never seen an infant, who has been treated in this manner, contract catarrhal troubles from an early bath, or have aught but healthy looking skins.

Greater care should be employed with children of unhealthy parentage, not only in the manner of bathing, but also other hygienic surroundings, and I firmly believe we can, by a little judicious early management, prevent a large per cent. of infant mortality.

\section{ECZEMA INFANTILE.}

Read before the Section of Diseases of Children, at the forty-third annual meeting of the American Medical Association, held at

BY B. MERRILL RICKETTS, M.D., OF CINCINNATI, 0 .

I believe that the subject "Eczema Infantile" will be of greater interest to this Section than any of the cutaneous diseases met during the first five years of childhood. It is a very common disease and one for which the general practitioner is frequently consulted. Not, however, until many of the numerous household remedies have proven themselves of no avail.

I do not care, on this occasion, to discuss the pathology or the cause from a bacteriological stand point. Neither do I care to report any special case or class of cases; only to speak of the eczema of childhood and its treatment, that we may discuss the management which has been the simplest, most easily applied with the best results thus far obtained by the author. There is not any skin disease that is so aggravating to childhood. There are none more unsightly. Usually both the head and face are involved. There are a few, where the disease is pretty generally distributed over the entire body and extremities.

When this is so, the child presents a most pitiful spectacle. The itching and discomfort occasioned by this condition are very aggravating and are sometimes accompanied by the most severe convulsive movements. There is nothing that appeals to a man's sympathy so much as one of these unfortunate conditions. I have seen those in charge of these unfortunate children, utterly oblivious to the suffering that the child endures.

The causes seem many, but to my mind there is none so common as that of the excessive use of soap and water.

My experience has been that about 95 per cent. of these cases are due to this cause. The disease is not: a respecter of persons, as it attacks the high and the low, the rich and the poor, and above all, the white skin races. The number of cases of this disease found among the dark akin races is proportionately very small. This perhaps, may be accounted for from the simple reason that the white skin is more delicate and easily soiled; hence the use of numerous cosmetics, which are so useless and injurious, and which are so extensively used. A clean faced child is the pride of every mother. She is lavish in the use of anything that will keep her child's face clean, usually the remedy that will remove the dirt the quickest. She uses these remedies more frequently with the thin, delicate skin of the child, than she does on the skin of her own face. The sebaceous glands should not exist if this law of extreme cleanliness holds good.

Nature is not responsible for children being reared in dirty atmospheres. These glands are for a purpose, namely to keep the skin constantly anointed with a fluid which protects the epithelium from becoming dry and fissured. That portion of the body which constantly should be anointed, is the one which is necessarily exposed to the wind, the rain, rays of the sun and dusts of various character, namely: the face and hands, which are forever exposed.

As the slime protects the epithelial structures of fish and mollusks, so does the sebaceous matter protect the cuticle of man. Birds anoint their feathers that they may be kept soft and pliable. Animals covered with hair, are likewise anointed by these glands, even the hairs themselves being supplied by oily secretion. The skin of the Mexican dog is rough, scaly and disagreeable to the touch. Not so with that of the hairy canine; his is oily and being so, enables him to endure more exposures to either the sun or rain, than his tropical ally. If we would but reflect, the cause of the disease, as a rule, could easily be determined, because having once been determined, the remedy is soon to be found. Not until mothers receive a certain degree of education in sanitary matters, can we expect a lessening in the number of these cases. At times the cause seems to be due to some nervous condition, while at other times there is indication that it is wholly local. The vesicular form, which is the most common, especially in infants, is obstinate and very trying to both the mother and child. After the disease has manifested itself, the child will begin to be irritable, restless, have loss 